\title{
Diabetes mellitus potentiates diffuse large B-cell lymphoma via high levels of CCL5
}

\author{
JINGCHENG ZHANG ${ }^{1}$, JIA LUO ${ }^{1}$, FANRONG LIU ${ }^{1}$, DONGMEI WU ${ }^{1}$, QINGLING ZHONG ${ }^{2}$, \\ LIANGTAO ZENG $^{1}$, ZIQING WU ${ }^{3}$, TONG ZHAO ${ }^{3}$, LIQING WU ${ }^{1}$ and HUA HAO ${ }^{1}$ \\ ${ }^{1}$ Department of Pathology, Second Affiliated Hospital, Nanchang University; ${ }^{2}$ College of Nursing, Medical College, \\ Nanchang University, Nanchang, Jiangxi 330006; ${ }^{3}$ Department of Pathology, Nanfang Hospital, \\ Southern Medical University, Guangzhou, Guangdong 510515, P.R. China
}

Received September 22, 2013; Accepted May 29, 2014

DOI: $10.3892 / \mathrm{mmr} .2014 .2341$

\begin{abstract}
There is much evidence suggesting that CCL5 is one of the chemoattractant cytokines involved in diabetes mellitus (DM) with diffuse large B-cell lymphoma (DLBCL). However, the pathological impact is unclear. In the current study, in order to improve understanding regarding the role of CCL5 in DM with DLBCL, the expression levels of CCL5 mRNA were examined in normal B cells, human DLBCL cell lines (Ly1, Ly8 and Ly10) and a mouse DLBCL cell line (A20), as well as those in cells cultured with either 5 or $30 \mathrm{mmol} / 1$ glucose. A20-CCL5 ${ }^{+}$(CCL5 overexpression) and A20-CCL5 (CCL5 knockdown) subclones were obtained through cell transduction with a lentiviral vector, and were subcutaneously injected into BALB/c DM mice and normal mice. Tumor growth was observed by calculating the tumor volume. The results demonstrated that CCL5 mRNA levels in DLBCL cells were significantly higher than those in the normal cells $(\mathrm{P}<0.05)$; and levels in DLBCL cells in $30 \mathrm{mmol} / \mathrm{l}$ Glu were significantly higher than in those of DLBCL cells in $5 \mathrm{mmol} / \mathrm{l}$ Glu $(\mathrm{P}<0.05)$. A20-CCL5 ${ }^{+}$cells led to tumor formation in DM mice compared with A20 and A20-CCL5- cells. These results indicate that high levels of CCL5 expression may accelerate DLBCL formation in DM.
\end{abstract}

\section{Introduction}

A study from 2008 suggested that the incidence of malignant tumors in individuals with diabetes mellitus (DM) is higher than that in those who do not suffer from DM (1). There is much evidence suggesting that CCL5 is one of the chemoat-

Correspondence to: Dr Fanrong Liu, Department of Pathology, Second Affiliated Hospital, Nanchang University, 1 Minde Road, Nanchang, Jiangxi 330006, P.R. China

E-mail: liufanrong@163.com

Key words: diabetes mellitus, CCL5, chemokine, diffuse large B-cell lymphoma tractant cytokines involved in DM with diffuse large B-cell lymphoma (DLBCL). However, the pathological impact is unclear (2).

CCL5 is a chemoattractant cytokine, and a number of studies have noted high levels of CCL5 expression in DM or indicated that DM is associated with CCL5 gene polymorphism $(3,4)$. Previous studies have demonstrated that the expression of CCL5 in DLBCL cells is upregulated by monocytes, and lymphoma may result from CCL5 reducing the ability of immunologic surveillance (5). Human DLBCL cell lines express CCL5 at high levels (6), and bioinformatic analyses suggest that CCL5 is an important cytokine involved in DM with DLBCL. Thus, it is essential to establish whether CCL5 accelerates DLBCL formation in DM.

The aim of the present study was to examine the expression of CCL5 mRNA in the A20 mouse DLBCL cell line for the first time to the best of our knowledge, by subcutaneously injecting A20-CCL5 ${ }^{+}$and A20-CCL5 ${ }^{-}$subclones into BALB/c $\mathrm{DM}$ mice and normal mice. A comparison of the tumor growth in these mice may improve the understanding of the role of CCL5 in DM with DLBCL.

\section{Materials and methods}

Materials. BALB/c mice ( $\mathrm{n}=90,6-8$ week-old males, weighing $25 \pm 3 \mathrm{~g}$ ) were purchased from the Central Laboratory of Animal Science of the Nanchang University Medical College (Nanchang, China). All animals received appropriate care according to the criteria outlined in the 'Guide for Care and Use of Laboratory Animals' [SYXK (gan) 2010-0002]. Normal B cells, human-derived DLBCL cell lines (Ly1, Ly8 and Ly10), and the BALB/c mouse-derived A20 DLBCL cell line were kindly provided by Professor Tong Zhao (Nanfang Hospital, Southern Medical University, Guangzhou, China).

Quantitative polymerase chain reaction ( $q P C R)$. All cell lines were cultured in RPMI-1640 medium with $10 \%$ heat-inactivated fetal bovine serum (FBS; HyClone, Logan, UT, USA), and were maintained in $37^{\circ} \mathrm{C}$ humidified cell culture incubators with $5 \% \mathrm{CO}_{2}$. The Ly1, Ly8, Ly10 and A20 cell lines were cultured in RPMI-1640 with 5 or $30 \mathrm{mmol} / \mathrm{l}$ glucose (Glu). Total RNA was extracted from cells using TRIzol reagent 
(Omega Bio-Tek Inc, Norcross, GA, USA) and the cDNA synthesis was performed using an RT-PCR kit (All-in-One ${ }^{\mathrm{TM}}$ qPCR mix; GeneCopoeia, Rockville, MD, USA) according to the manufacturer's instructions. PCR primers were purchased from Invitrogen (Carlsbad, CA, USA) with the following sequences: hCCL5: F, 5'-CGCTGTCATCCTCATTGCTA-3'; and R, 5'-GCACTTGCCACTGGTGTAGA-3'. GAPDH: F, 5'-ACAGTCAGCCGCATCTTCTT-3'; and R, 5'-GACAAG CTTCCCGTTCTCAG-3'. mCCL5: F, 5'-CCCTCACCATCA TCCTCACT-3'; and R, 5'-CTTCTTCTCTGGGTTGGCAC-3'. SDHA: F, 5'-AGGTATCAATGCTGCTCTGG-3'; and R, 5'-AAGTAGGTTCGCCCGTAG-3'. All reactions were performed in triplicate. The results were analyzed using Image-Pro Plus software, version 6.0 (IPP; Media Cybernetics, Rockville, MD, USA).

Cell transduction. 293T human embryonic kidney (HEK293T) cells were maintained in Dulbecco's modified Eagle's medium (DMEM; Solarbio, Beijing, China) culture solution with $10 \%$ FBS. The murine leukemia cell line A20 (B lymphocytic) was cultured in RPMI-1640 medium supplemented with $10 \%$ FBS and $0.05 \mathrm{mM} 2$-mercaptoethanol. pLKO-TRC short hairpin RNA (shRNA) clones (TRCN0000068102, TRCN0000068101, TRCN0000068100, TRCN0000068099 and TRCN0000068098) targeting mouse CCL5 were purchased from Open Biosystems (Huntsville, AL, USA). cDNA encoding human CCL5 was synthesized and cloned into a pBABE retroviral vector (Cell Biolabs , Inc., San Diego, CA, USA). For cell transduction, retroviruses were prepared by transient co-transfection with helper viral vector into HEK293T cells using calcium phosphate precipitation (Macgene Biotechnology Ltd., Beijing, China). HEK293T cells were transfected with plasmid DNA and cultured at $37^{\circ} \mathrm{C}$ for $6 \mathrm{~h}$, then the medium was replaced with RPMI-1640 medium supplemented with 10\% FBS. At 36-48 h post-transfection, the supernatant was collected and filtered through a $0.45-\mu \mathrm{m}$ filter. Cells were infected at a density of $\sim 2 \times 10^{6}$ per $10-\mathrm{cm}$ cell culture dish in DMEM and RPMI-1640 (50:50) supplemented with $8 \mu \mathrm{g} / \mathrm{ml}$ polybrene (Solarbio). After $24 \mathrm{~h}$, the medium was changed to RPMI-1640 supplemented with $10 \%$ FBS and $0.05 \mathrm{mM}$ 2-mercaptoethanol and cells were cultured for further assay. Cell transduction with the lentiviral vector encoding the shRNA that targets mouse CCL5 was conducted following the manufacturer's instructions (Open Biosystems). Western blotting and qPCR were performed to confirm the overexpression or knockdown, and consequently identify the A20-CCL5 ${ }^{+}$and A20-CCL5 subclones, respectively.

Animals and in vivo tests. All procedures were conducted under sterile conditions, and the animal protocol for this experiment was approved by the Animal Care and Use Committee of the Nanchang University Medical College. The $90 \mathrm{BALB} / \mathrm{c}$ mice were randomly divided into two groups of 45 : The DM models and the controls. DM models were established with intraperitoneal injection of streptozocin according to a previous method $(7,8)$ and randomized into an A20-CCL5 ${ }^{+}$, A20-CCL5 and A20 group. Each group also existed in the normal mice (Table I). Tumor cells $\left(2 \times 10^{7}\right)$ in $0.2 \mathrm{ml}$ growth medium (RPMI-1640 medium supplemented with $10 \%$ FBS)
Table I. Assignment of animals into groups.

\begin{tabular}{lll}
\hline Group & Mouse type & Cells inoculated \\
\hline A1 & DM & A20-CCL5 $5^{+}$ \\
A2 & DM & A20-CCL5 \\
A3 & DM & A20 \\
B1 & Normal & A20-CCL5 $5^{+}$ \\
B2 & Normal & A20-CCL5 \\
B3 & Normal & A20 \\
\hline
\end{tabular}

$\mathrm{n}=15$ in each group. DM, diabetes mellitus.

were subcutaneously injected into the left axillary fossa of each mouse. Tumor growth was measured by calculating the tumor volume. Mice were sacrificed by cervical dislocation 30 days post-injection. Blood serum was collected to detect secreted CCL5 protein by ELISA and a skin incision was made to remove the tumor.

Immunohistochemistry. Tissue specimens were collected from tumors in the mice, fixed in formalin and embedded in paraffin (Solarbio). The tissues were then cut into $3-\mu \mathrm{m}$ sections and dried on capillary-gap glass slides (Solarbio). Immunohistochemistry of the paraffin sections was performed using the ChemMate ${ }^{\mathrm{TM}}$ EnVision $^{\mathrm{TM}}{ }^{\mathrm{T}}$ Detection kit (Dako, Carpinteria, CA, USA). The sections were dewaxed, dehydrated, subjected to antigen retrieval and blocked (Solarbio) with hydrogen peroxide for endogenous peroxidase activity. The sections were then immunostained with CCL5 antibody (brown color) with DAB substrate (Dako), and counterstained using hematoxylin and eosin (Solarbio). Image analysis was accomplished to measure the average integral optical density of the positive cells using IPP software, version 6.0 (9).

ELISA. The concentration of mCCL5 in the blood serum was measured using commercially available kits (KHC1031; Invitrogen) according to the manufacturer's instructions. The range of sensitivity for mCCL5 was $0-2,000 \mathrm{pg} / \mathrm{ml}$. The assay was performed according to the manufacturer's instructions. ELISA plates were assessed at $450 \mathrm{~nm}$ using Curve Expert software (Hyams Development, Chattanooga, TN, USA).

Statistical analysis. SPSS software version 20.0 (IBM, Armonk, NY, USA) was used for statistical analyses. Numerical values are expressed as the mean \pm standard deviation. Statistical significance was calculated using the Student's $\mathrm{t}$-test for paired samples. $\mathrm{P}<0.05$ was considered to indicate a statistically significant difference.

\section{Results}

hCCL5 mRNA expression in different cell lines with normal culture. The mRNA expression of hCCL5 in normal B and DLBCL cell lines was examined by qPCR. All the three DLBCL cell lines (Ly1, Ly8 and Ly10) expressed CCL5 mRNA (Fig. 1A). Compared with those in the normal B cells, 


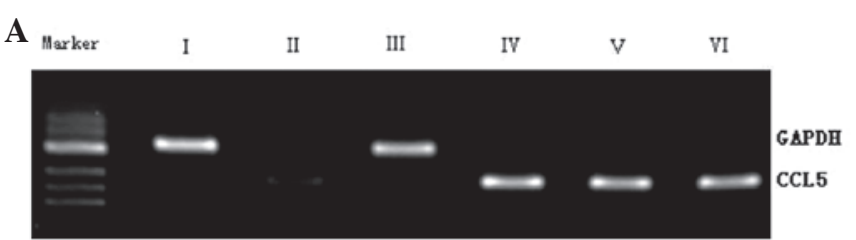

B

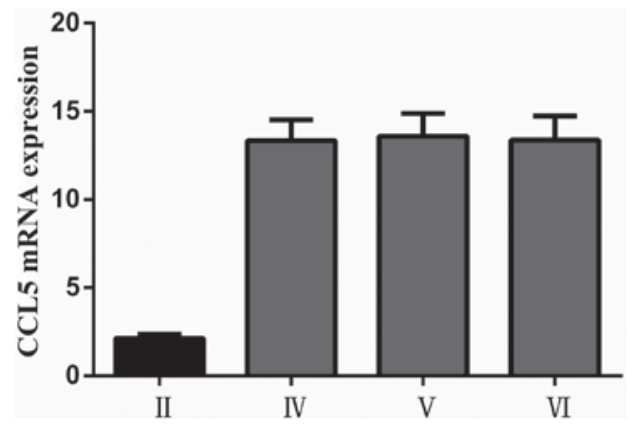

Figure 1. Densitometric analysis of the expression of the CCL5 gene in B cell and DLBCL cell lines. (A) Expression of CCL5 mRNA assessed by qPCR. (B) Graph representing relative expression levels of CCL5 mRNA based on qPCR results ("P<0.05). Lanes: I and III, Control; II, B cell; IV, Ly1; V, Ly8; and VI, Ly10. DLBCL, diffuse large B-cell lymphoma; qPCR, quantitative polymerase chain reaction.

the CCL5 mRNA expression levels in the three cell lines were increased significantly $(\mathrm{P}<0.05$; Fig. 1B).

CCL5 mRNA expression in different cell lines with 5 or $30 \mathrm{mmol} / \mathrm{l} \mathrm{Glu}$ culture. All cell lines were collected following culture in 5 or $30 \mathrm{mmol} / \mathrm{l}$ Glu for 2 weeks. The CCL5 mRNA expression in the cell lines was examined by qPCR. All the human cell lines (Ly1, Ly8 and Ly10) expressed hCCL5 mRNA(Fig. 2A), and the A20 cells expressed mCCL5 mRNA (Fig. 2C). Compared with those in the cells cultured with $5 \mathrm{mmol} / \mathrm{l} \mathrm{Glu}$, the CCL5 mRNA expression levels in the cell lines cultured with $30 \mathrm{mmol} / \mathrm{l}$ Glu were increased significantly ( $\mathrm{P}<0.05$; Fig. 2B and D).

Histology of tumors in BALB/c mice. Subcutaneous tumor models in BALB/c mice were successfully established. Tumor growth of the DM groups (A1-3) was higher than that of the normal mouse groups (B1-3, respectively) (Fig. 3). The time frames of tumorigenesis in the DM mice (A1-3) were shorter than those in the normal mouse groups (B1-3). Mice were sacrificed 30 days after injection and the skin was dissected to reveal the tumors. Tumors were round or oval, and the tumor surface had a nodular appearance. They were adherent to the skin of the capillary-rich surface, were firm and tan-white, and presented no distant lymph node transfers. The DM CCL5 ${ }^{+}$(A1) group tumor volumes were significantly greater than those in the DM control group (A3) $(\mathrm{P}<0.01)$, but there was no significant difference in tumor volume between $\mathrm{CCL}^{+}$overexpression and control groups in the normal mice (B1 and B3) ( $\mathrm{P}=0.729)$ (Table II). Using a microscope, it was possible to observe the size of the tumor cells in the tumor tissues. The cells had large nuclei that were irregular in size and shape and the nuclear staining was uneven, with scant cytoplasm. A small number of pathological mitotic figures and small lymphocytes were observed. With hematoxylin and eosin staining, the A20 tumors were observed to have
A

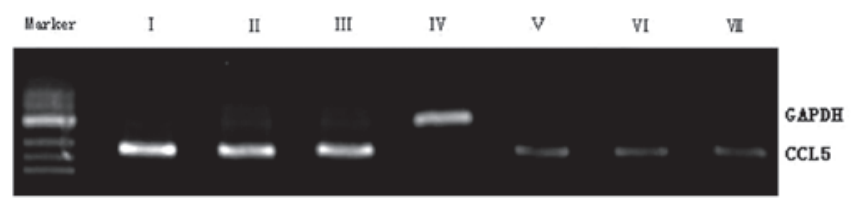

B

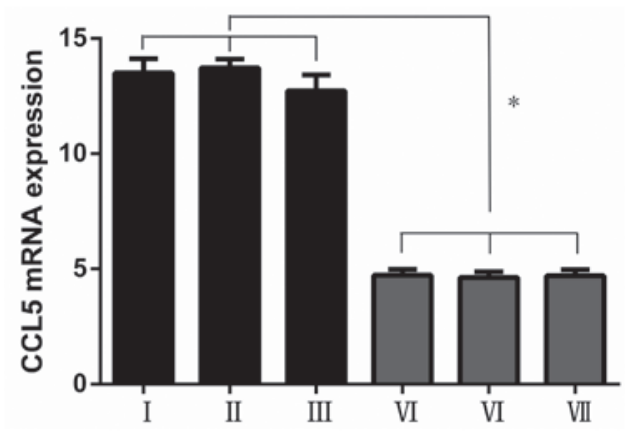

C

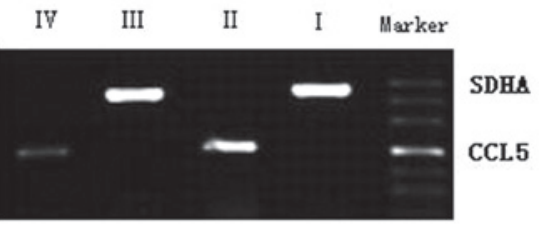

D

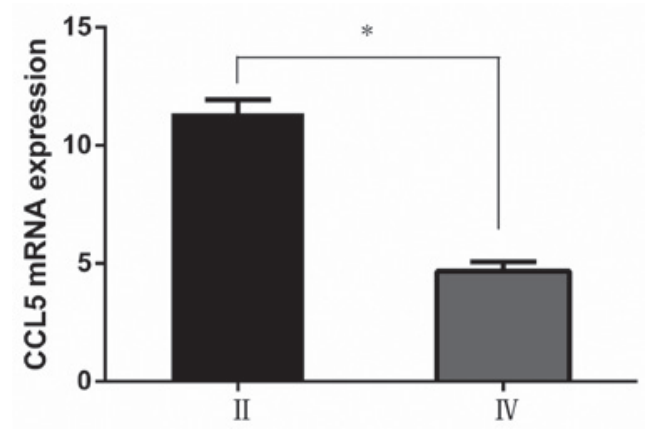

Figure 2. Densitometric analysis of the expression of the CCL5 gene in DLBCL and A20 cell lines cultured in high and low sugar concentrations. (A) Expression of CCL5 mRNA assessed by qPCR in DLBCL cells. (B) Graph representing relative expression levels of CCL5 mRNA assessed by qPCR $(\mathrm{P}<0.05)$. Lanes: I-III, $30 \mathrm{mmol} / 1$ Glu-cultured DLBCL cells; IV, control; V-VII, $5 \mathrm{mmol} / \mathrm{l}$ Glu-cultured DLBCL cells. All assays were performed in triplicate. (C) Expression of CCL5 mRNA assessed by qPCR in A20 cells. (D) Graph representing relative expression levels of CCL5 mRNA assessed by qPCR ("P<0.05). Lane: I and III, control; II, $30 \mathrm{mmol} / 1$ Glu-cultured A20 cells; IV, 5 mmol/1 Glu-cultured A20 cells. All assays were performed in triplicate. DLBCL, diffuse large B-cell lymphoma; qPCR, quantitative polymerase chain reaction.

diffuse homogeneous infiltrate consisting of large and cohesive tumor cells with moderate cytoplasm and pleomorphic nuclei (Fig. 4A). Immunohistochemical staining results demonstrated that the expression levels of CCL5 in DM mouse cells were higher those in normal mouse cells, and $\mathrm{CCL}^{+}$mouse cells displayed more staining than CCL5 ${ }^{-}$cells (Fig. 4).

DM and normal serum expression levels of CCL5 protein. Serum samples were collected from each group, and expression levels of the CCL5 protein in the serum were measured by ELISA. The results demonstrated that diabetic BALB/c mice secreted significantly higher levels of soluble CCL5 protein compared with those of normal mice $(\mathrm{P}<0.05)$. CCL5 ${ }^{+}$tumor 
Table II. Tumor growth of DM and normal BALB/c mouse groups following A20 cell injection.

\begin{tabular}{lllrrrrr}
\hline Group & Mouse type & Cells inoculated & $\mathrm{n}$ & $\%$ & Time (day) & Weight $(\mathrm{g})$ & ${\text { Volume }\left(\mathrm{mm}^{3}\right)}^{\text {(n) }}$ \\
\hline A1 & DM & A20-CCL5 & 14 & 93.3 & $7.0 \pm 0.85$ & $2.11 \pm 0.42$ & $1612.40 \pm 191.94^{\mathrm{a}}$ \\
A2 & DM & A20-CCL5 & 9 & 60.0 & $9.5 \pm 2.80$ & $1.12 \pm 0.14$ & $998.00 \pm 99.85^{\mathrm{a}}$ \\
A3 & DM & A20 & 10 & 66.6 & $9.0 \pm 1.80$ & $1.67 \pm 0.22$ & $1367.20 \pm 139.25$ \\
B1 & Normal & A20-CCL5 & 3 & 20.0 & $12.0 \pm 1.30$ & $1.33 \pm 0.42$ & $1030.00 \pm 343.95^{\mathrm{b}}$ \\
B2 & Normal & A20-CCL5 & 3 & 20.0 & $14.0 \pm 2.50$ & $1.25 \pm 0.40$ & $970.00 \pm 274.18^{\mathrm{b}}$ \\
B3 & Normal & A20 & 7 & 46.6 & $12.0 \pm 4.20$ & $1.30 \pm 0.43$ & $1025.83 \pm 237.74$
\end{tabular}

Volume and weight measured 30 days after the injection. ${ }^{\mathrm{a}} \mathrm{P}<0.01$ vs. A3 group, ${ }^{\mathrm{b}} \mathrm{P}=0.729$ vs. B3 group. DM, diabetes mellitus.

Table III. CCL5 protein levels in the peripheral blood of different groups (pg/ml).

\begin{tabular}{|c|c|c|c|c|}
\hline Group & Normal with tumor & Normal no tumor & DM with tumor & DM no tumor \\
\hline A20-CCL5 ${ }^{+}$ & $118.07 \pm 1.33^{\mathrm{b}}$ & $88.11 \pm 5.32$ & $223.78 \pm 3.78^{a}$ & $180.84 \pm 3.98$ \\
\hline A20-CCL5 & $121.69 \pm 2.91^{\mathrm{b}}$ & $62.34 \pm 3.42$ & $98.25 \pm 3.05^{\mathrm{a}}$ & $147.54 \pm 3.78$ \\
\hline A20 & $106.64 \pm 3.14$ & $79.23 \pm 3.67$ & $179.40 \pm 3.42$ & $159.55 \pm 3.90$ \\
\hline
\end{tabular}

All experiments were performed in triplicate. $\mathrm{P}<0.05$, DM mice vs. normal mice, ${ }^{\mathrm{a}} \mathrm{P}<0.01 \mathrm{vs}$. DM with normal CCL5 expression, ${ }^{\mathrm{b}} \mathrm{P}=0.138$ vs . normal mice with normal CCL5 expression. DM, diabetes mellitus.

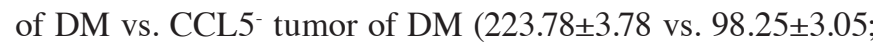
$\mathrm{P}<0.01) \mathrm{CCL}^{+}$tumor of normal vs. CCL5- tumor of normal (118.07 \pm 1.33 vs. $121.69 \pm 2.91 ; \mathrm{P}=0.138)$ (Table III).

\section{Discussion}

Data from the current study demonstrated that CCL5 expression levels in high-sugar cultivated human and mouse DLBCL are higher than those of cells cultured in low-sugar medium. ELISA detection indicated that protein levels of CCL5 in DM mouse BALB/c serum are higher than those in normal mice. This may be due to a complicated mechanism of secretion of CCL5, which is caused by high blood sugar. However, according to previous studies there is a close association between diabetes and the impaired islet function; and lack of islet function is associated with the chemical absorption of CCL5 by lymphocytes (10). It has also been demonstrated that long-term insulin resistance can directly promote the secretion of CCL5 in the body (11). On the other hand, the high sugar levels in the serum of patients with DM may lead to elevated CCL5 levels in the body, which are associated with the expression of MCP-1, TNF- $\alpha$, IL-6 and AGE. This implies that the body exhibits different degrees of inflammation and that the chemokine CCL5 is an important factor in inflammation (12). In addition, the risk of diabetes has been demonstrated to be closely associated with the levels of CCL5 protein in the body; and increased levels of CCL5 may directly lead to a greater risk of suffering from diabetes (13).

In the present study, CCL5 expression levels in human DLBCL cell lines were demonstrated to be significantly higher than those in normal B cells. CCL5 may be involved in the occurrence and development of tumors, and a number of studies have reported that it is secreted in tumor tissue by tumor cells or inflammatory cells. CCL5 is also important in the occurrence and development of malignant tumors, as it can be involved in the growth of blood vessels in the tumor tissues (14). Another study indicated that CCL5 may directly affect tumor cells through paracrine signaling and promote the growth of the tumor, so the majority of tumor cells can directly secrete CCL5 (15). CCL5 can also adjust the expression and secretion of normal T cells. For example, CCL5 at the tumor site can promote the formation of tumor blood vessels and biological activity by aggregating and activating matrix metalloproteinases, in particular MMP-9, in tumor cells in order to increase the rate of growth and metastasis of tumors (16).

Diabetes itself is one of the inducing factors of malignant tumors (1), as the high blood glucose levels lead to the production of a large number of free radicals and induce the accumulation of reactive oxygen species (ROS). Excessive ROS production can promote multiple glycolytic pathways and produce $\mathrm{AGE}$ and $\mathrm{PkG}$, which cause DNA damage and mutations and may result in malignant tumor growth (17). Insulin resistance can easily lead to an increase in existing high insulin levels observed in diabetes; and this build-up can speed up the growth of tumors (18-20). As a result, the present study established A20 cell lines with different levels of CCL5 expression by slow virus transfection in order to identify whether CCL5 is important in diabetes with malignant tumors. The animal tumor models demonstrated that the DM tumor mice transfected with the CCL5-overexpressing A20 cell line presented higher CCL5 protein levels in the peripheral blood than those of the control mice. The average maximum tumor volume and weight of DM mice was greater than that of the normal mice, suggesting that high blood sugar levels in the 
A

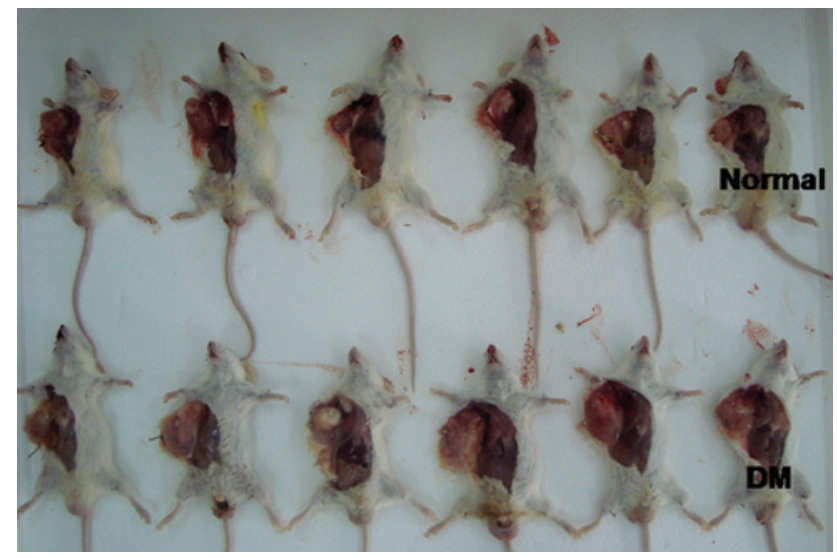

C

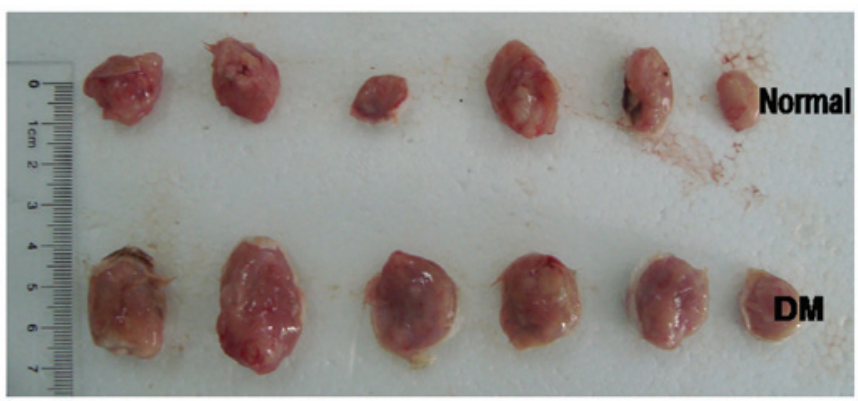

B

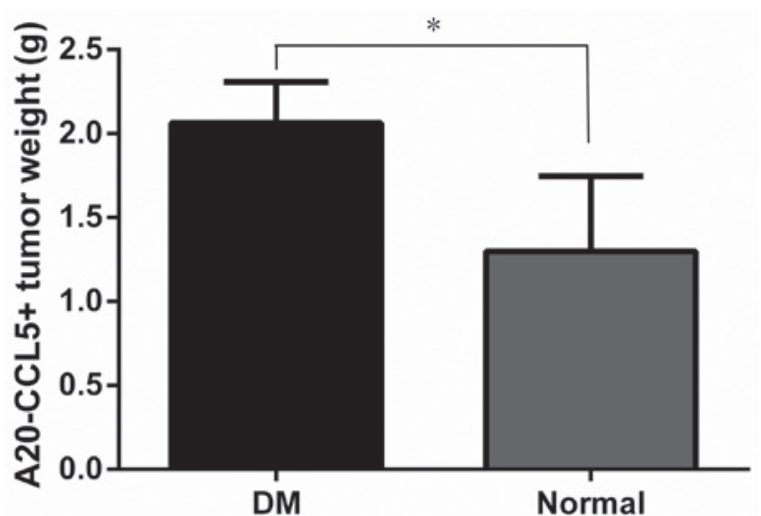

D

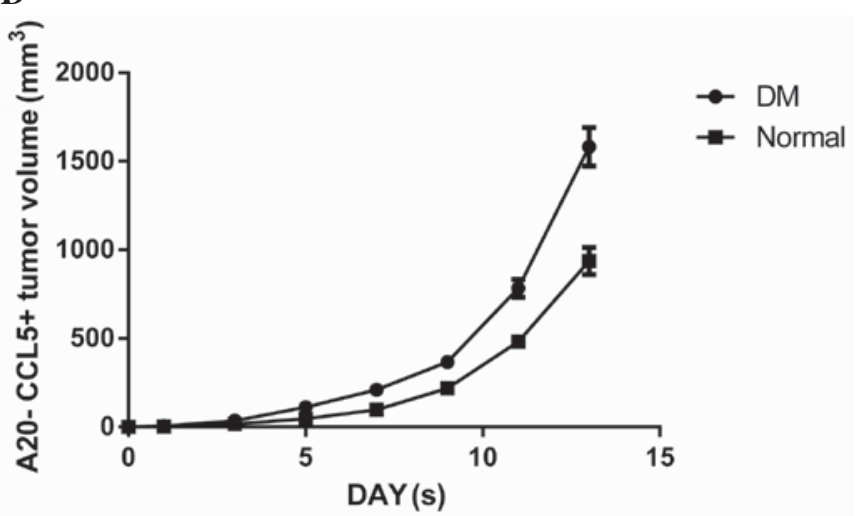

Figure 3. Tumor growth in normal and DM mice transfected with CCL5-overexpressing A20 cells. (A and C) Images of tumors following sacrifice. Comparison of tumor growth in DM and normal mice, represented by (B) final tumor weight and (D) tumor volume. Mean tumor volume was measured at the start of injection of A20 cells after 10 days. The bars represent the mean \pm standard deviation (" $\mathrm{P}<0.05)$. DM, diabetes mellitus.
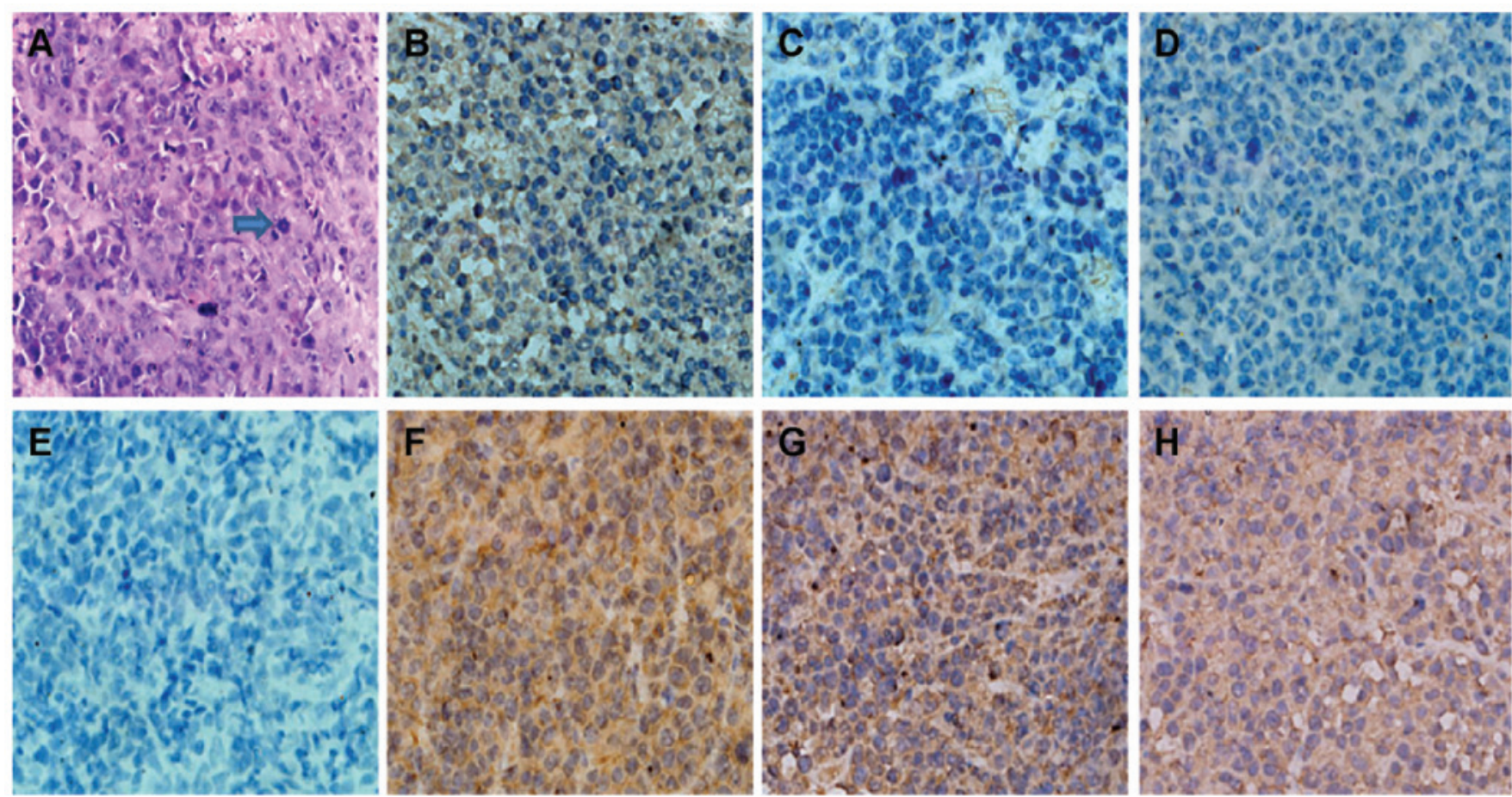

Figure 4. Representative images of the expression patterns of CCL5 in different tumor tissues. (A) HE staining of tumors from DM mice following inoculation with normal A20 cells. CCL5 immunohistochemical staining of sections of normal mouse tumor tissues following inoculation with (B) A20 cells with CCL5-overexpression, (C) A20 cells with CCL5 knockdown and (D) normal A20 cells. (E) Control staining, without CCL5 antibody. (F) CCL5 immunohistochemical staining of sections of DM mouse tumor tissues following inoculation with A20 cells with CCL5-overexpression, (G) A20 cells with CCL5 knockdown and $(\mathrm{H})$ normal A20 cells. The arrow refers to an abnormal diffuse large B cell. Magnification, x400. HE, hematoxylin and eosin; DM, diabetes mellitus. 
body can increase the occurrence and potentiate the development of DLBCL. There was no significant difference in the tumor growth capacity of the normal mouse model (group B3) and normal mouse model with CCL5 knockdown (group B2). These results indicate that the knockdown of CCL5 in DM may reduce and slow tumor growth, but this effect of CCL5 knockdown is not observed in the normal, non-diabetic mice. Therefore, this experiment indicates a key role for the chemokine CCL5 in the diabetic mouse with DLBCL.

Diabetes decreases immunity, and the occurrence of DLBCL has a complex association with immune function; DLBCL is more likely to occur on the condition that overall immune function decreases. Nevertheless, a large number of background inflammatory cells infiltrate the DLBCL tumor tissue, suggesting that the immune response occurs during the development of DLBCL to a certain extent. Various studies have demonstrated that inflammatory cells can be induced by certain chemokines (21), and CCL5 in the body has been noted to attract $\mathrm{T}$ helper 2 and $\mathrm{T}$ regulatory cells into tumor and adjacent tissues (22-24). In DLBCL, CCL5 triggers $\mathrm{T}$ cells to release interleukin 2 and to promote the occurrence of DLBCL (25). CCL5 can weaken the immune surveillance function in the body, increasing the probability of DLBCL occurrence (26). Additionally, the low immunity combined with high CCL5 levels may lead to the occurrence of DLBCL. The current study demonstrated that CCL5 is important in the induction of DLBCL in DM mice. Diabetes increases the levels of CCL5 protein in the body and in return, the high expression levels of CCL5 accelerate the growth, invasion and metastasis of tumors. The BALB/c mouse genome is $>90 \%$ homologous to humans, so mouse tumors present similarities to those of humans with respect to organization, formation and developmental processes (27). Therefore, the present study may provide reliable theoretical basis for the study of DLBCL in human diabetes. Unfortunately, in the current study, it was not possible to collect a sufficient number of tissue samples from patients with DM combined with DLBCL, thus no analysis of human samples was conducted. In future, it will be valuable to compare the CCL5 expression of DLBCL tumor tissues of diabetic and non-diabetic patients in order to provide further evidence that CCL5 is key in diabetic human patients with DLBCL. Thus, the present study provides novel insight for the prevention and control of DLBCL in DM.

\section{Acknowledgements}

The current study was supported by the National Natural Science Foundation of China (grants 81160251, 81101434 and 81071659).

\section{References}

1. Renehan AG, Tyson M, Egger M, et al: Body-mass index and incidence of cancer: a systematic review and meta-analysis of prospective observational studies. Lancet 371: 569-578, 2008.

2. Mitri J, Castillo J and Pittas AG: Diabetes and risk of Non-Hodgkin's lymphoma: a meta-analysis of observational studies. Diabetes Care 31: 2391-2397, 2008.

3. Nakajima K, Tanaka Y, Nomiyama T, et al: RANTES promoter genotype is associated with diabetic nephropathy in type 2 diabetic subjects. Diabetes Care 26: 892-898, 2003.
4. Herder C, Peltonen M, Koenig W, et al: Systemic immune mediators and lifestyle changes in the prevention of type 2 diabetes: results from the Finnish Diabetes Prevention Study. Diabetes 55: 2340-2346, 2006.

5. Lehmann MH, Schreiber S, Vogelsang H and Sigusch HH: Constitutive expression of MCP-1 and RANTES in the human histiocytic lymphoma cell line U-937. Immunol Lett 76: 111-113, 2001.

6. Liu F, Zhang Y, Wu ZQ and Zhao T: Analysis of CCL5 expression in classical Hodgkin's lymphoma L428 cell line. Mol Med Rep 4: 837-841, 2011.

7. Liu FR, Zhong QL, Liu DW, et al: Establishment of type 1 diabetic $\mathrm{BALB} / \mathrm{c}$ mouse models and detection of $\mathrm{CD} 4^{+} \mathrm{CD} 25^{+}$regulatory T cells. Guangdong Med J 32: 427-429, 2011 (In Chinese).

8. Zhong QL, Liu FR, Liu DW, et al: Expression of $\beta$-catenin and cyclin D1 in epidermal stem cells of diabetic rats. Mol Med Rep 4: 377-381, 2011.

9. Kraan MC, Smith MD, Weedon H, et al: Measurement of cytokine and adhesion molecule expression in synovial tissue by digital image analysis. Ann Rheum Dis 60: 296-298, 2001.

10. Carvalho-Pinto C, García MI, Gómez L et al: Leukocyte attraction through the CCR5 receptor controls progress from insulitis to diabetes in non-obese diabetic mice. Eur J Immunol 34: 548-557, 2004.

11. Bogdanski P, Pupek-Musialik D, Dytfeld J, et al: Influence of insulin therapy on expression of chemokine receptor CCR5 and selected inflammatory markers in patients with type 2 diabetes mellitus. Int J Clin Pharmacol Ther 45: 563-567, 2007.

12. Ando H, Kurita $\mathrm{S}$ and Takamura T: The specific p38 mitogen-activated protein kinase pathway inhibitor FR167653 keeps insulitis benign in nonobese diabetic mice. Life Sci 74: 1817-1827, 2004.

13. Nakamura K, Yamagishi S, Adachi H et al: Serum levels of sRAGE, the soluble form of receptor for advanced glycation end products, are associated with inflammatory markers in patients with type 2 diabetes. Mol Med 13: 185-189, 2007.

14. Kulbe H, Levinson NR, Balkwill F and Wilson JL: The chemokine network in cancer - much more than directing cell movement. Int J Dev Biol 48: 489-496. 2004.

15. Soria $\mathrm{G}$ and Ben-Baruch A: The inflammatory chemokines CCL2 and CCL5 in breast cancer. Cancer Lett 267: 271-285, 2008.

16. Adler EP, Lemken CA, Katchen NS and Kurt RA: A dual role for tumor-derived chemokine RANTES (CCL5). Immunol Lett 90: 187-194, 2003.

17. Brownlee M: The pathobiology of diabetes complications: a unifying mechanism. Diabetes 54: 1615-1625, 2005.

18. Goodwin PJ, Ennis M, Pritchard KI, et al: Fasting insulin and outcome in early-stage breast cancer: results of a prospective cohort study. J Clin Oncol 20: 42-51, 2002.

19. Saydah SH,Loria CM, Eberhardt MS and Brancati FL: Abnormal glucose tolerance and the risk of cancer death in the United States. Am J Epidemiol 157: 1092-1100, 2003.

20. Richardson LC and Pollack LA: Therapy insight: Influence of type 2 diabetes on the development, treatment and outcomes of cancer. Nat Clin Pract Oncol 2: 48-53, 2005.

21. Mueller CG, Boix C, Kwan WH, et al: Critical role of monocytes to support normal B cell and diffuse large B cell lymphoma survival and proliferation. J Leukoc Biol 82: 567-575, 2007.

22. Skinnider BF and Mak TW: The role of cytokines in classical Hodgkin lymphoma. Blood 99: 4283-4297, 2002.

23. Aldinucci D, Lorenzon D, Cattaruzza L, et al: Expression of CCR5 receptors on Reed-Steinberg cells and Hodgkin lymphoma cell lines: involvement of CCL5/Rantes in tumor cell growth and microenvironmental interactions. Int J Cancer 122: 769-776, 2008.

24. Fischer M, Juremalm M, Olsson N, et al: Expression of CCL5/RANTES by Hodgkin and Reed-Sternberg cells and its possible role in the recruitment of mast cells into lymphomatous tissue. Int J Cancer 107: 197-201, 2003.

25. Appay V and Rowland-Jones SL: RANTES: a versatile and controversial chemokine. Trends Immunol 22: 83-87, 2001.

26. Sun N, Yang G, Zhao H, et al: Multidose streptozotocin induction of diabetes in BALB/c mice induces a dominant oxidative macrophage and a conversion of THl to $\mathrm{TH} 2$ phenotypes during disease progression. Mediators Inflamm 2005: 202-209, 2005.

27. Bernardi R, Grisendi S and Pandolfi PP: Modelling haematopoietic malignancies in the mouse and therapeutical implications. Oncogene 21: 3445-3458, 2002. 